TITLE:

$<$ NEWS>International symposium to celebrate 40 years of research on the Taï chimpanzee

$\operatorname{AUTHOR}(S)$ :

Nakamura, Michio

CITATION:

Nakamura, Michio. <NEWS>International symposium to celebrate 40 years of research on the Taï chimpanzee. Pan Africa News 2019, 26(1): 2-3

ISSUE DATE:

2019-06

URL:

http://hdl.handle.net/2433/245229

RIGHT:

Copyright (C) Pan Africa News. 


\title{
International symposium to celebrate 40 years of research on the Taï chimpanzee
}

\author{
Michio Nakamura \\ Graduate School of Science, Kyoto University, Japan \\ (E-mail: nakamura@jinrui.zool.kyoto-u.ac.jp)
}

An international symposium to celebrate 40 years of the wild chimpanzee study project at Taï Forest in Côte d'Ivoire (see Wittig 2018) was held on May 29-31, 2019 at the Max Planck Institute for Evolutionary Anthropology, Leipzig, Germany (Figure 1). The symposium also commemorated the retirement of Dr. Christophe Boesch, who initiated the research project at Taï, and has served as Director of the Department of Primatology at the Max Planck Institute (see Boesch 2009: 2012; Boesch \& Boesch-Achermann 2000 for some of Dr. Boesch's achievements). Approximately 40 speakers from Germany, Switzerland, USA, UK, and Japan, and guests from Côte d'Ivoire were invited to the symposium. Many students from the Max Planck Institute and other institutions, who are studying wild chimpanzees, were also in attendance.

On May 29, the symposium began with the session "Field Site Comparison," chaired by Dr. Roman Wittig. Dr. Wittig, together with Drs. Cat Hobaiter, Anne Pusey, Dave Morgan, Alexander Piel, Takeshi Furuichi, and Martin Muller, introduced their study sites in Taï, Budongo (Uganda), Gombe (Tanzania), Goalougo (Congo), Issa (Tanzania), Kalinzu (Uganda), and Kanyawara (Uganda). At the conclusion of the first day, Dr. Boesch gave a commemorative lecture to reflect on 40 years of research at the Taï Chimpanzee Project (Figure 2).

On the morning of the second day, the "Field Site Comparison" session continued. Drs. Simone Pika, Michio Nakamura, and David Watts spoke about their field sites at Loango (Gabon), Mahale (Tanzania), and Ngogo (Uganda). At the end of the session, Dr. Hjalmar Kühl introduced the Pan African Project, which analyzes dozens of wild chimpanzee study sites for direct behavioral comparisons.

The subsequent session on second day, entitled "Communication," was chaired by Dr. Klaus Zuberbühler. In this session, Drs. Catherine Crockford and Katie Slocombe presented vocalization studies conducted at Taï and Budongo. Dr. Julia Fischer presented her work concerning vocalization studies in green monkeys. The "Cognition" session followed, which was chaired by Dr. Robert Seyfarth. In this session, Drs. Cedric GirardButtoz and Erica van de Waal presented their field experimental studies of cognition in chimpanzees and vervet monkeys, respectively, while Dr. Daniel Haun presented his studies of human cognition. The last session on the second day, "Cooperation," was chaired by Dr. John Mitani. In this session, Drs. Liran Samuni, Kevin

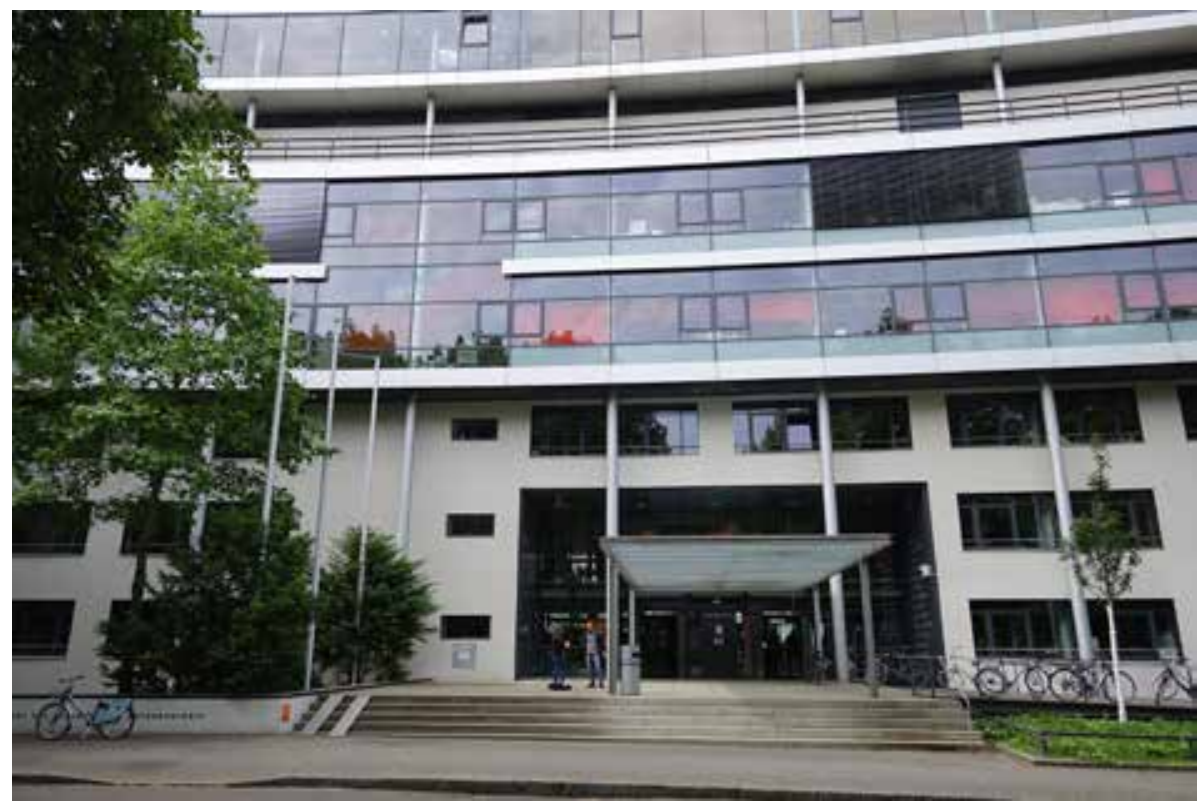

Figure 1. The Max Planck Institute for Evolutionary Anthropology, Leipzig 


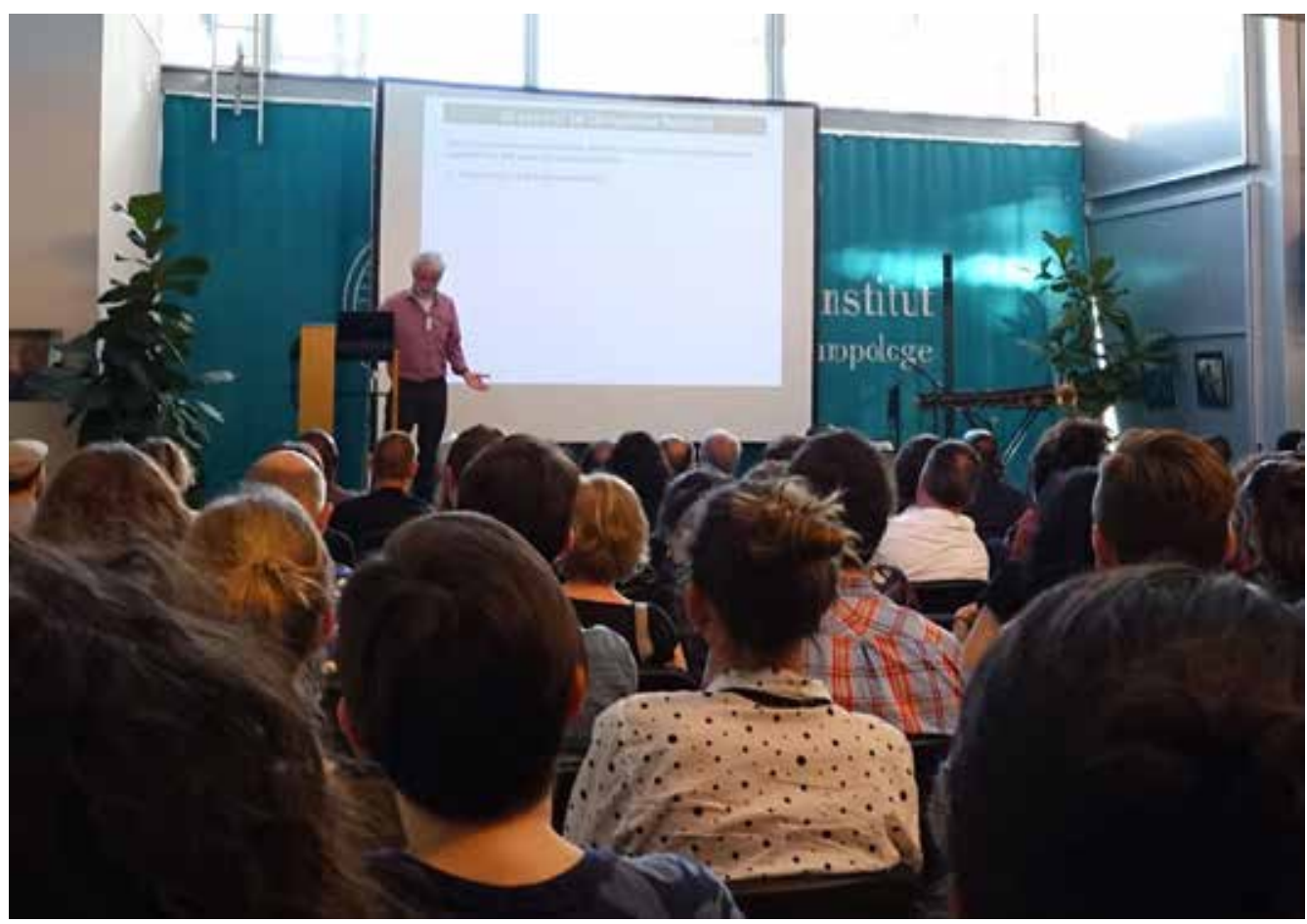

Figure 2. Dr. Christophe Boesch giving a commemorative lecture on May 29

Langergraber and Redouan Bshary discussed collaborative behavior in Taï chimpanzees, Ngogo chimpanzees, and vervet monkeys, respectively.

On May 31, Dr. Richard Wrangham chaired the session "Competition." Drs. Roman Wittig and Michael Wilson discussed within- and inter-group competition among Taï and Gombe/Kanyawara chimpanzees, respectively. Dr. Julia Lehmann presented her work concerning the role of friendship when competition becomes severe among Barbary macaques. Finally, Richard McElreath presented his work concerning competition among humans in different cultures. Dr. Christophe Boesch chaired "Culture," the second session on the third day. Drs. Lydia Luncz, Crickette Sanz, and Caroline Schüppli presented their studies of material culture in Taï and Goualougo chimpanzees, and in orangutans, respectively. Dr. Ignacio de la Torre presented work concerning early human stone technology relative to current stone tool use by nonhuman primates. The final session concerned great ape physiology, genetics, and health, and was chaired by Dr. Jacinta Beehner. In this session, Drs. Tobias Deschner and Melissa Emery Thompson discussed the physiology of Taï and Kanyawara chimpanzees, respectively. Dr. Linda Vigilant presented her genetic research in the Taï chimpanzees, and Dr. Fabian Leendertz presented work concerning great ape health and disease. After these sessions concluded, Dr. Carel van Schaik summarized the symposium by introducing the outcomes of the questionnaires participants completed during the symposium.
Overall, the symposium was well-organized, and was an excellent learning and networking experience with prominent primate researchers across the globe. Amazingly, most of these eminent scientists are studying the common topic of chimpanzees in the wild. Notably, "big data" of behavioral and genomic studies on chimpanzees is now available from several long-term study sites. Such big data, if re-interrogated with new ideas, will provide new insights into our cousin species in the African forests.

Finally, I should note that, as proposed by Drs. Boesch and Wittig, the participants signed an appeal for the conservation of wild chimpanzees, and a press release was made during the meeting.

\section{REFERENCES}

Boesch C 2009. The Real Chimpanzee: Sex Strategies in the Forest. Cambridge University Press, Cambridge.

Boesch C 2012. Wild Cultures: A Comparison between Chimpanzee and Human Cultures. Cambridge University Press, Cambridge.

Boesch C, Boesch-Achermann H 2000. The Chimpanzees of the Taï Forest: Behavioral Ecology and Evolution. Oxford University Press, Oxford.

Wittig RM 2018. 40 years of research at the Taï Chimpanzee Project. Pan Afr News 25:16-18. 